\title{
Appareil
}

\section{Notations pour la guitare électrique}

\section{Martin Laliberté}

\section{(2) OpenEdition}

Journals

Édition électronique

URL : http://journals.openedition.org/appareil/1017

DOI : 10.4000/appareil.1017

ISSN : 2101-0714

\section{Éditeur}

MSH Paris Nord

\section{Référence électronique}

Martin Laliberté, « Notations pour la guitare électrique », Appareil [En ligne], 5 | 2010, mis en ligne le 09 juin 2010, consulté le 30 juillet 2020. URL : http://journals.openedition.org/appareil/1017 ; DOI : https://doi.org/10.4000/appareil.1017

Ce document a été généré automatiquement le 30 juillet 2020.

\section{(c) (i) (2) $\Theta$}

Appareil est mis à disposition selon les termes de la Licence Creative Commons Attribution - Pas d'Utilisation Commerciale - Pas de Modification 4.0 International. 


\title{
Notations pour la guitare électrique
}

\author{
Martin Laliberté
}

\section{Introduction}

1 La musique pour guitare électrique contemporaine bénéficie depuis plus de trente ans d'un vocabulaire sonore et gestuel très développé : son propre "classique ", son sale blues-rock, sons extravagants électroacoustiques, sons méticuleux spectraux... Noter ces effets inventés par les musiciens depuis 1940 pose des défis considérables et provoque des recherches passionnantes, comme le montrent les travaux des participants de ce colloque.

2 Pour contribuer à une analyse critique de cette musique et de sa notation, cet article procède en deux temps asymétriques. Le premier effectue un bref rappel des archétypes que je propose et leurs incidences sur le geste musical. Le second est consacré à une étude illustrée de la notation pour guitare électrique, en relation avec ces modèles.

\section{Gestes et archétypes}

\section{1. Archétypes instrumentaux et musicaux}

3 En amont de l'étude des gestes de la guitare électrique, il faut remonter à certains principes. Comme je l'ai proposé dans plusieurs publications antérieures ${ }^{1}$, l'étude de la musique sur une vaste période permet de constater la présence de courants de fond influençant les attentes des musiciens, des compositeurs et des facteurs d'instruments de musique. On peut qualifier ces modèles $d$ '« attracteurs » ou d'« archétypes » :

- la voix, idéalisation de la voix humaine ;

- la percussion, cristallisation du geste musical ;

- les deux archétypes génèrent un espace mixte, véritable centre d'intérêt de cette façon d'aborder la musique. 
Le modèle vocal est une idéalisation de la voix humaine et de ses voyelles. Il se caractérise principalement par la continuité, la tendance à l'harmonicité et favorise l'expression mélodique, horizontale. Cet archétype s'incarne souvent dans le chant mais aussi, et c'est ici qu'il rend service, dans la musique purement instrumentale ou électronique. L'imitation de la voix est un idéal dès le début de la musique instrumentale, comme en témoignent les traités baroques occidentaux ou bon nombre de traditions non-européennes. À la limite, l'archétype vocal atteint la sinusoïde éternelle, parfaitement dénuée d'intérêt musical.

5 Le modèle percussif est l'expression de la tendance complémentaire, issue du geste manuel, de la frappe sur un objet sonore ou de la consonne. Il privilégie le momentané, le discontinu, la complexité, l'inharmonicité ou le vertical. Cet archétype concerne les instruments de percussions mais aussi bon nombre d'aspects de tous les autres instruments et de la voix chantée ${ }^{2}$. Sa limite, toute aussi absurde que celle du pôle complémentaire, est le bruit blanc continu, un changement permanent.

6 Ces deux modèles attirent les développements des instruments de musique, en particulier lors des périodes de mutations technologiques (fig. 1) :

Figure 1 : vocalisation des guitares

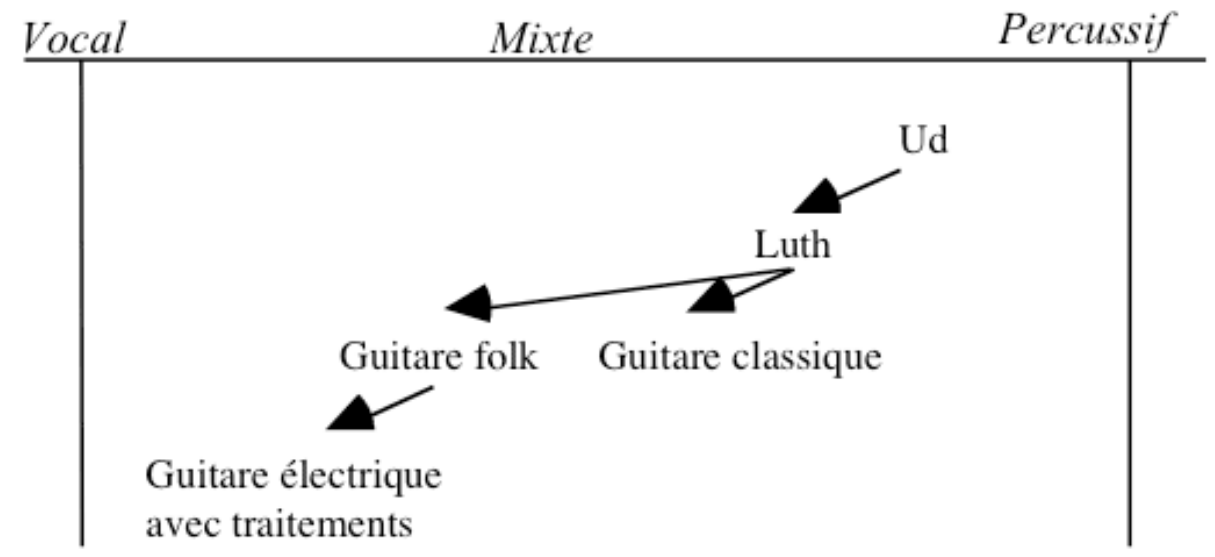

7 Les deux limites étant réellement plus des asymptotes que des idéaux - en cela ce paradigme n'est pas du tout platonicien ni jungien - le véritable lieu d'intérêt est l'espace mixte. L'étude de la part percussive dans un instrument plutôt vocal, par exemple dans la voix chantée, ou de la part vocale dans un instrument percussif, par exemple un piano, se révèle utile. Voilà aussi pourquoi les termes acoustiques usuels (son entretenu, son résonant) ne recouvrent pas complètement ce qui est proposé et pourquoi un autre vocabulaire est devenu nécessaire.

8 L'espace manque pour compléter ces démonstrations. Le lecteur intéressé peut aussi consulter mes autres travaux ${ }^{3}$.

\section{1. 1. Typologie des gestes musicaux}

9 Ces modèles se révèlent utiles pour une étude du geste musical. On peut en effet classer les principales familles de gestes musicaux dans l'espace mixte (fig. 2) : 
Figure 2 : gestes et archétypes

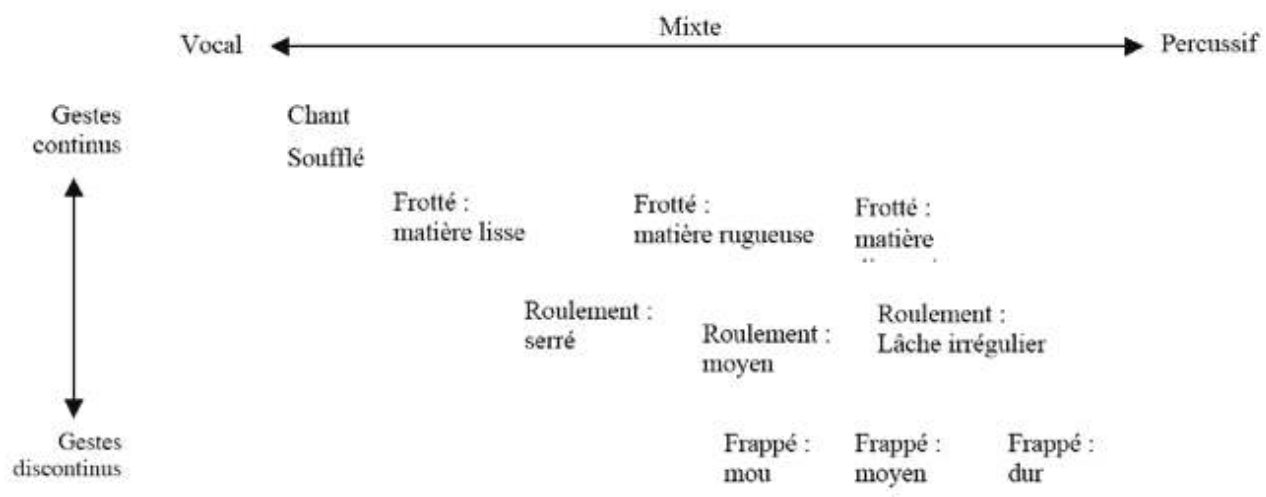

10 On le voit, les gestes s'approchent de l'un ou l'autre modèle en fonction de leur continuité relative mais aussi en fonction des qualités des matières en présence ${ }^{4}$. Ces qualités de gestes ont une influence directe sur le son mais aussi sur l'écriture de la musique, point focal de cet article.

\section{1. 2. Étude des gestes de la guitare électrique : Jimi à Woodstock}

11 Les notations de la musique pour guitare électrique sont directement dépendantes des sons recherchés et des gestes nécessaires. Étudions rapidement la gestique particulière du guitariste de la maturité complète de l'instrument, lors de sa célèbre improvisation autour du Star Spangled Banner à Woodstock en 1969.

Une typologie des principaux gestes d'Hendrix se dégage (fig. 3) :

Figure 3 : gestes de Jimi Hendrix, Star Spangled Banner, 1969

\begin{tabular}{|c|c|c|}
\hline Geste & Son associé & Commentaire \\
\hline Mouvements de bouche a & $\begin{array}{l}\begin{array}{l}\text { Mouvements de la pédale } \\
\text { wahh-wah }\end{array} \\
\end{array}$ & $\begin{array}{l}\text { «Voyelles » spécifíques et } \\
\text { trajets formantiques }\end{array}$ \\
\hline Mouvements de bouche $b$ & $\begin{array}{l}\text { Mouvements mélodiques } \\
\text { précis, jeu normal }\end{array}$ & $\begin{array}{l}\text { Gestes mélodiques, souvent } \\
\text { improvisés }\end{array}$ \\
\hline Mouvements de bouche c & $\begin{array}{l}\text { Mouvements mélodiques } \\
\text { précis, jeu bend mg }\end{array}$ & Pour la justesse et le contrôle \\
\hline Mouvements de bouche d & $\begin{array}{l}\text { Mouvements mélodiques } \\
\text { tige whanmy }\end{array}$ & De même, à plus grande échelle \\
\hline Mouvements de bouche e & Glissandos sur le manche & Sorte d'ornementation \\
\hline Mouvements de bouche $f$ & Phrasés dynamiques & $\begin{array}{l}\text { Plus rares : phrasé surtout dans } \\
\text { le jeu au médiator }\end{array}$ \\
\hline Mouvements de langue a & Trilles et trémolos & Geste exagéré, volontaire? \\
\hline Langue $b$ & Jeu sur les cordes & Effet spectaculaire (son mou) \\
\hline $\begin{array}{l}\text { Mouvements de pieds/jambes } \\
\text { a }\end{array}$ & Jeu sur pédales continues & Pour wah-wah et volume \\
\hline Trémolo de pied & Sur wah-wah & Effet de cliquetis \\
\hline $\begin{array}{l}\text { Mouvements de pieds/jambes } \\
\text { b }\end{array}$ & $\begin{array}{l}\text { Jeu sur commutateurs } \\
\text { discontinus }\end{array}$ & $\begin{array}{l}\text { Distorsion, compresseur et Mu- } \\
\text { Tron }\end{array}$ \\
\hline Coups de pieds dans le vide & $\begin{array}{l}\text { Ponctuation sonore ou } \\
\text { respiration }\end{array}$ & \begin{tabular}{|l} 
Distincts des mouvements de \\
pedales fonctionnelles
\end{tabular} \\
\hline Main gauche sur tige whammy & Glissandi prononcés & \\
\hline $\begin{array}{l}\text { Main gauche sur les boutons } \\
\text { rotatifis }\end{array}$ & $\begin{array}{l}\text { Ajustements de volume ou } \\
\text { brillance }\end{array}$ & $\begin{array}{l}\text { Dose les dégradés (plus } \\
\text { finement qu' avec le pied) }\end{array}$ \\
\hline $\begin{array}{l}\text { Main gauche sur le } \\
\text { commulateur de micros a }\end{array}$ & Couleur de base & $\begin{array}{l}\begin{array}{l}\text { Sombre (accords), mixte, brillant } \\
\text { (solo) }\end{array} \\
\end{array}$ \\
\hline $\begin{array}{l}\text { Main gauche sur le } \\
\text { commutateur de micros b }\end{array}$ & Trémolo de commutateur & Effetet de cliquetis \\
\hline Mains frappant les cordes & Explosions et bruits & Bruits à plus grande échelle \\
\hline Mains frottant les cordes & Glissandi inharmoniques & Bruits glissants \\
\hline $\begin{array}{l}\text { Balancements de la main } \\
\text { droite }\end{array}$ & Bends et vibratos & $\begin{array}{l}\text { Génération des effets. } \\
\text { À remarquer trois vibratos } \\
\text { différents : transversal, } \\
\text { perpendiculaire et par } \\
\text { secouement de lindex } \\
\text { Phrasé des vibratos }\end{array}$ \\
\hline
\end{tabular}


Figure 4 : gestes d'Hendrix et archétypes

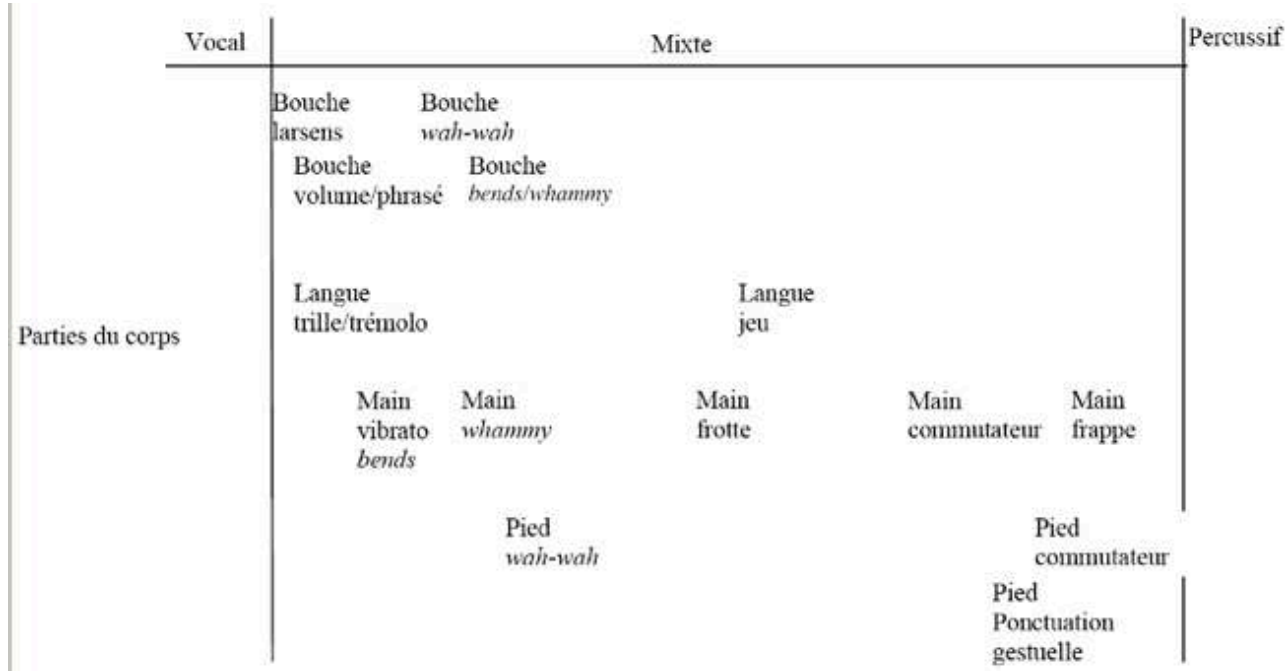

15 De telles différences de gestes et de fonctions musicales entraînent des besoins de notations complémentaires et poussent les notations de guitare électrique vers une inventivité intéressante.

\section{Guitare électrique et notations}

En effet, cette notation a connu des phases contrastées qui ont peu à peu constitué l'usage courant, par dessus lequel viendront se greffer d'autres méthodes découlant de la musique savante contemporaine.

\section{1. Notations commerciales : à la recherche de la précision}

17 La musique pour guitare électrique se note principalement par des moyens traditionnels, voire archaïques, mais avec des degrés de précision divers. Deux morceaux de Jimi Hendrix vont nous servir d'exemple : Foxy Lady et Purple Haze.

\section{1. 1. Foxy Ladies}

Cette transcription pose des premiers jalons (fig. 5) ${ }^{6}$ : 
Figure 5 : Foxy Lady 1: notation simple (1983)

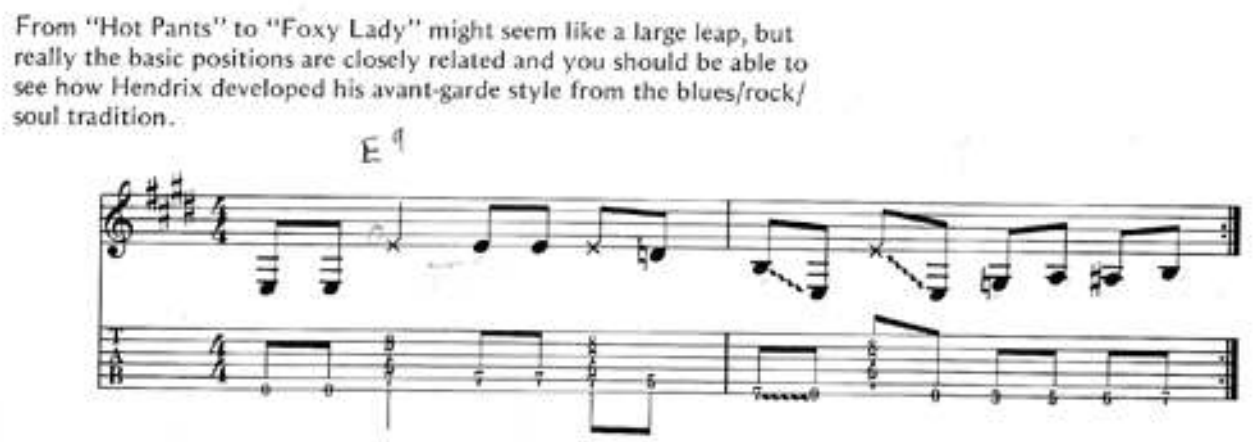

Dans cette notation assez sommaire, l'esprit du morceau est transcrit dans ses grandes lignes, mais plusieurs approximations demeurent. Le ton de Mi est simple, mais ne correspond pas au ton réel du morceau (Fa\# modal). Par ailleurs, l'harmonie n'est pas explicitée mais représentée par un « $\mathrm{x}$ » dans la notation solfégique. Rythmiquement, cette transcription est aussi grandement simplifiée : les petits contretemps et syncopes joués sont égalisés pour faciliter la lecture ${ }^{7}$. Enfin, la tablature, résurgence frappante des pratiques renaissantes ou baroques, apparaît assez nettement comme la partition principale, tandis que la notation solfégique est un complément, peut-être même une concession pour faire « sérieux $»^{8}$.

Voyons une seconde version, plus élaborée (fig. 6) ${ }^{9}$ :

Figure 6 : Foxy Lady 2: notation plus correcte

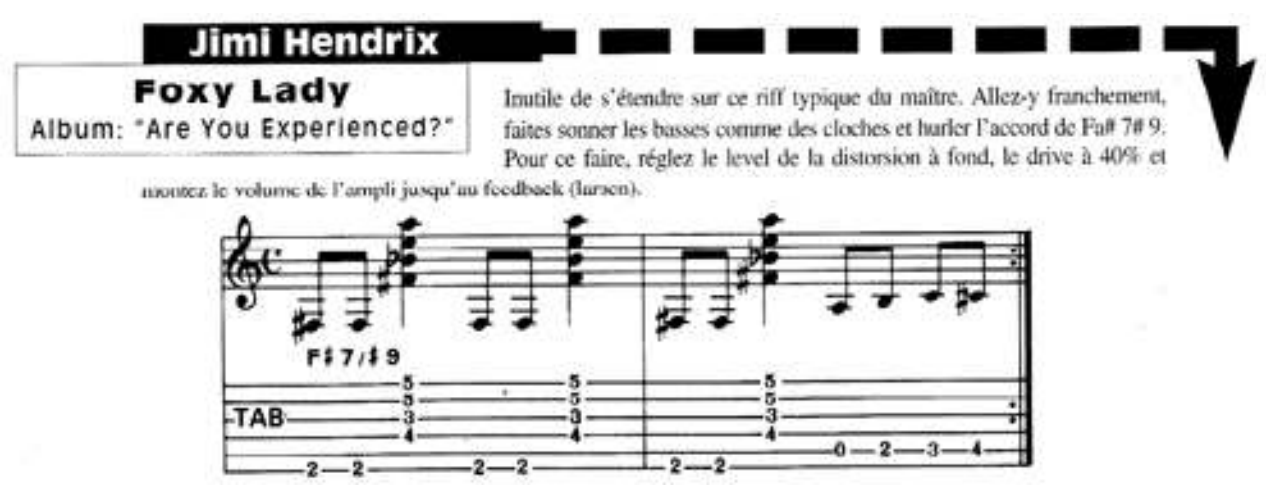

Ce second cas est plus satisfaisant: le ton implicite est juste et l'harmonie utilement indiquée. Par contre, la notation rythmique demeure trop simple et l'armure incorrecte. On devine encore la prépondérance de la tablature sur la notation solfégique. Remarquons enfin les commentaires pertinents pour l'esprit du morceau et sa sonorité.

La troisième transcription se veut nettement plus ambitieuse; elle représente l'état actuel des « bonnes » transcriptions (fig. 7) ${ }^{10}$ : 
Figure 7 : Foxy Lady 3: notation détaillée

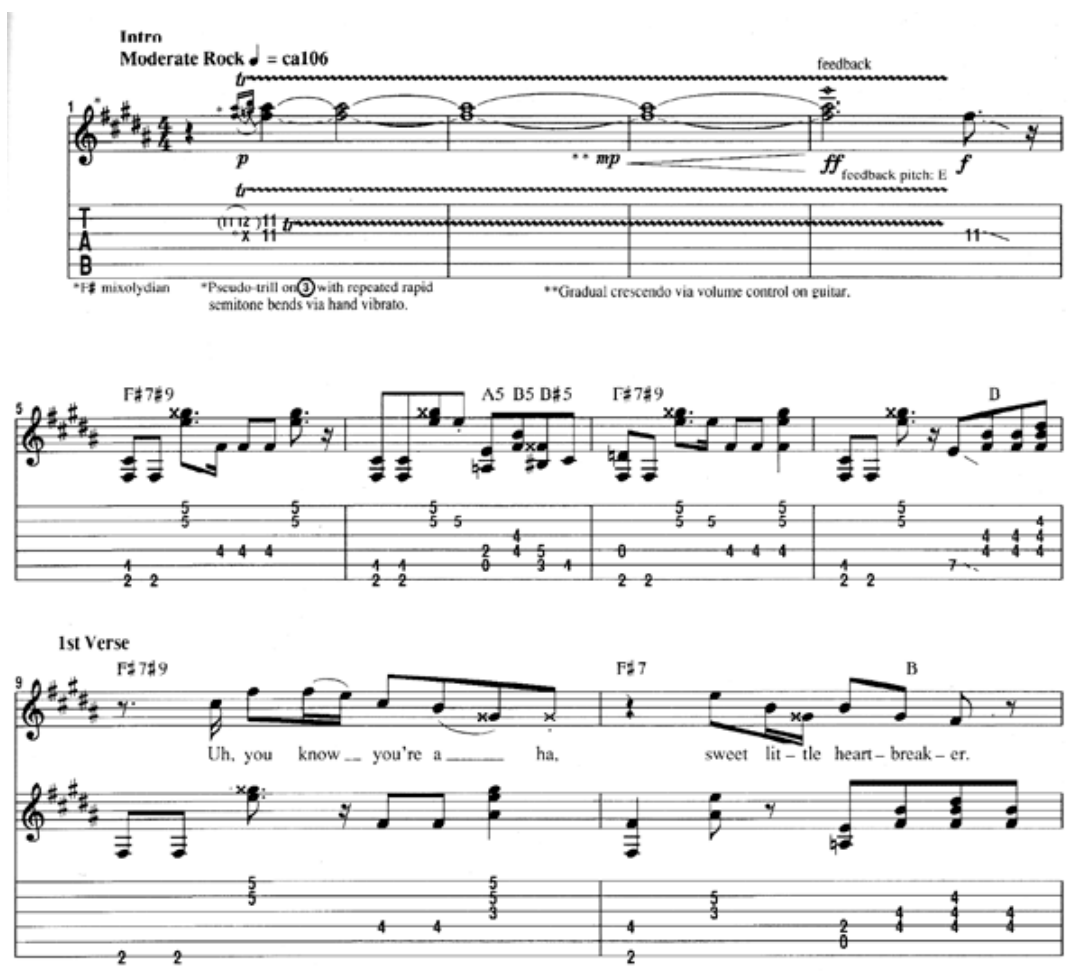

23 Ce recueil soigné tente de "tout " noter: effets de timbre, fluctuations rythmiques, partie vocale, et même la basse et la batterie dans des parties séparées. Cette notation détaillée se révèle complète et satisfaisante pour la musique d'Hendrix, en complément d'une écoute de l'enregistrement. La transcription non seulement des notes, tempo, rythmes, harmonie et mode mais aussi de plusieurs effets de timbre (larsen, pseudo trille, contrôle de volume) se révèle intéressante. Pour arriver à ce résultat, les éditeurs Bella Godiva et Hal Léonard ont réuni une équipe de spécialistes, dont un musicologue pour les notices analytiques et l'héritier musical d'Hendrix, Alan Douglas. Certains problèmes se posent encore pourtant.

\section{1. 2. Quelle notation pour Purple Haze?}

Ce second morceau phare d'Hendrix est similaire, mais permet d'aller un peu plus loin.

Voyons d'abord l'introduction au morceau :

In this rendition ${ }^{11}$ of his own classic Jimi remains pretty faithful to the original. There are some exceptions, including subtle melodic variations in the bridge solo, insertion of several lead fills in the verses, and replacement of the fade-out at the song's conclusion with a recapitulation of the intro theme. Unlike the bridge solo, this theme is all within the confines of the E minor/ 'blues' pentatonic scale (E G A B D).

Just in the first five measures of the bridge solo there are three distinct scalar sources that can be isolated. Starting with the first measure, here the temporary center is A and the repeated four-note guitar riff can be traces directly to the major pentatonic scale (A B C\# E F\#). The second measure finds him playing a similar pattern, but now he's returned to the E minor/ 'blues' pentatonic scale and continues doing so into the next measure. The remainder of the solo is based on the E Dorian mode (E F\# G A B C\# D) and, as you'll see, most of the positions used are the same as ones commonly relied on to play the E minor/ 'blues' pentatonic scale, 
often referred to colloquially as 'boxes'.

As he did in 'Love or Confusion', Jimi used the thumb in conjunction with the fingers for fretting chords throughout 'Purple Haze'. For example, he frets the root of the A major (A C\# E) on the sixth string with his thumb ${ }^{12}$.

\section{modales et mélodiques.}

Comme dans le cas de Foxy Lady, la notation se révèle précise et pratique (fig. 8) ${ }^{13}$ :

Figure 8 : Purple Haze: transcription détaillée
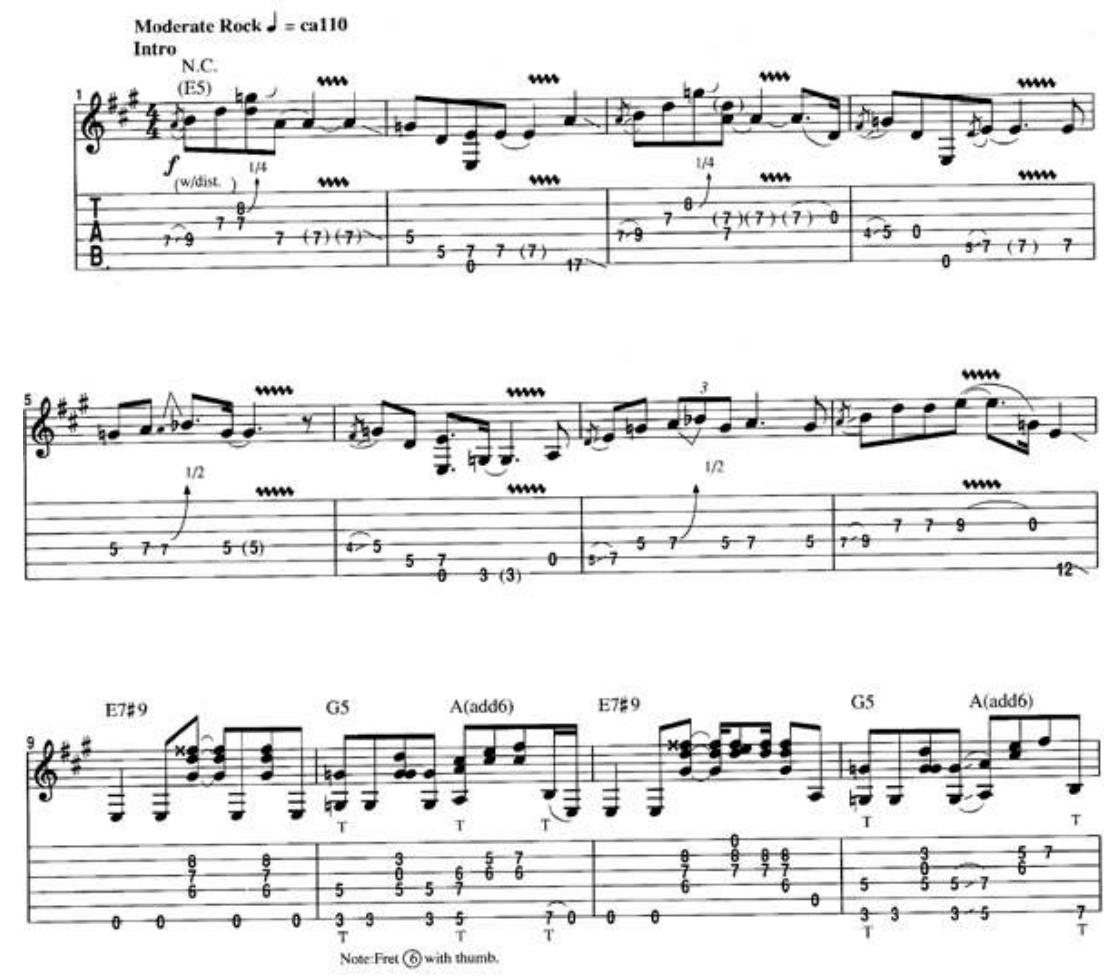

On peut toutefois se poser la question de la fonction d'une telle transcription. Les nombreuses nuances indiquées avec rigueur dépassent souvent le niveau « nécessaire ». Comme on le sait bien, à l'intérieur de ce genre musical, il y a davantage de fluctuations interprétatives, ce fameux "groove», tout aussi difficile à cerner que l'ancien swing ou même la notion de blues, que d'interprétation littéralement exacte, il n'y a qu'à comparer les différentes versions en concert de ce morceau par son compositeur. Tant de précision est-elle bien nécessaire ${ }^{14}$ ? Par ailleurs, les nombreuses finesses rythmiques compliquent la lecture pour le guitariste moyen, dont le réflexe sera plutôt de ne lire que la tablature, quitte à " groover » à l'oreille ou " au feeling " au moment de jouer le morceau. La situation est encore plus nette dans d'autres passages plus complexes de ce morceau, notamment le solo du pont auquel se réfère la notice ou d'autres plus virtuoses encore, provoquant des pages aussi «noires» de notes que celles de Zappa ou, peut-être Ferneyhough.

Dans ce contexte, une notation simplifiée devient peut-être moins réductrice qu'il n'y paraissait de prime abord (fig. 9) ${ }^{15}$ : 
Figure 9 : Purple Haze: une notation aussi efficace?

\section{Jimi Hendrix}

\section{Album: "Are You Experienced?"}

Une composition géniale qui a tellement compté pour la guitare et la musique en général. Comment une telle magie a-t-elle pu émaner d'un son de guitare? Le fait de dénicher une vieille fuzz au Germanium vous aidera. sinon: drive à $80 \%$, level à $40 \%$ et peu de médiums. Pour la magie, désolé, on ne l'achète pas dans un magasin de musique!
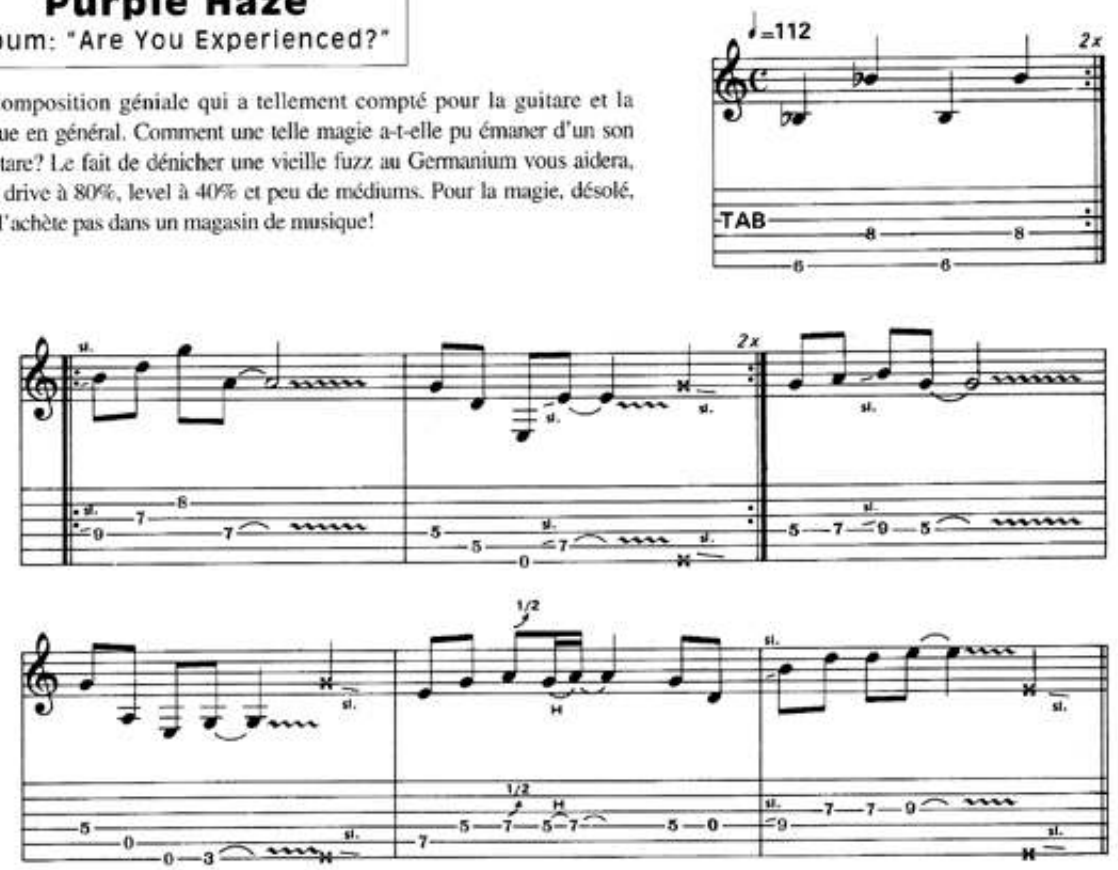

Simple, cette transcription est jouable et facilement interprétable au sens fort du terme. La guitare rock retrouve ici la pratique solidement établie des jazzmen et leur realbook: une transcription simple mais pertinente pour une musique dont la part orale et interprétative demeure la plus importante. Il faut toutefois ajouter que le recueil cidessus ne transcrit que ce fragment.

\section{1. 3. Symboliques standardisées}

La transcription de la musique de guitare électrique existe depuis plus de cinquante ans, si on inclut le jazz, et elle a pris bon nombre d'habitudes. Voici la nomenclature des symboles la plus complète, celle des éditions Hal Leonard (fig. 10) ${ }^{16}$ : 
Figure 10 : nomenclature détaillée
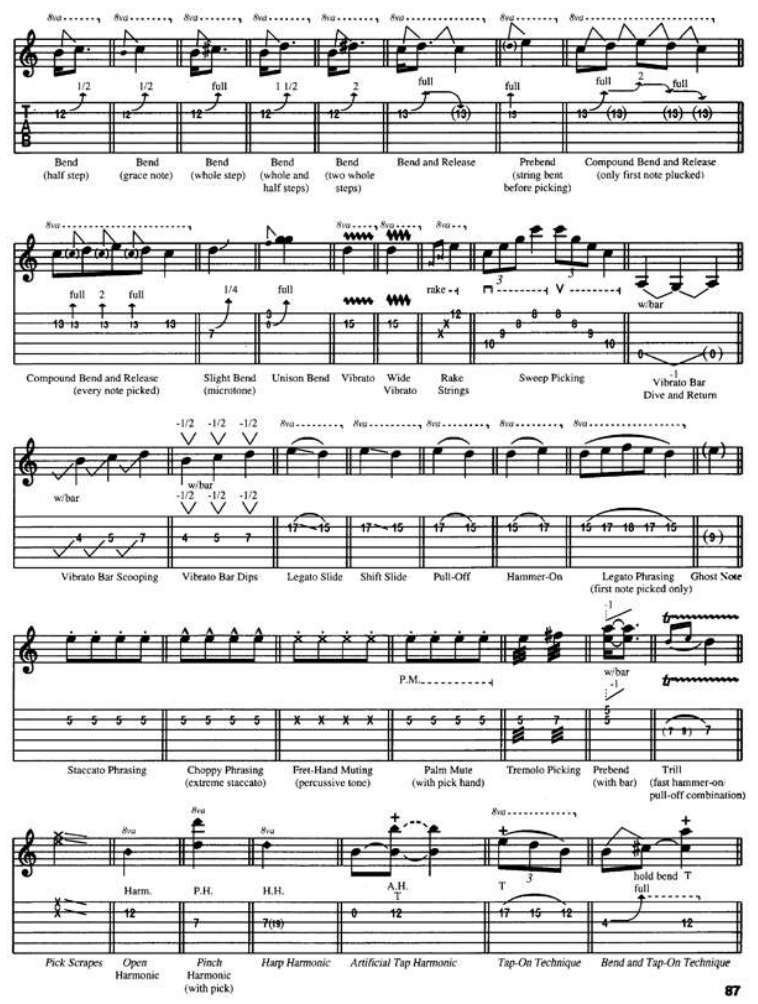

31 La plupart des techniques usuelles de main droite et gauche s'y retrouvent, avec une notation efficace et désormais stabilisée. Dans ce tableau, le temps semble d'ailleurs s'écouler du haut vers le bas, des bends microtonaux du blues, aux principales techniques du Heavy Metal et aux techniques à deux mains des années 1980-1990. Cela dit et à nouveau, quel guitariste lit vraiment cela ? La part d'apprentissage demeure ici très orale ou intuitive.

\section{1. 4. Bilan de la notation commerciale}

En phase avec les éléments musicaux primordiaux de ces musiques - ou du moins avec sa théorisation dominante -, la notation commerciale de la guitare électrique rock est essentiellement une notation mélodico-rythmique traditionnelle.

Un examen plus attentif révèle toutefois des éléments plus importants qu'il n'y paraît de prime abord:

- renaissance de la tablature baroque, modernisée par une rythmique moderne et une précision graphique ;

- résolution très idiomatique des difficultés de doigté et de timbres des cordes ;

- introduction des micro-intervalles issus du blues voire des musiques de studio (blue-notes, $1 / 4$ de tons ou intervalles non-spécifiés). Cela tend déjà vers le continuum des hauteurs contemporain, a fortiori si l'on emploie un whammy bar ou un bottleneck;

- notation simple d'effets de timbres : réglages de pédales d'effets, volume, trilles et vibratos variés, effets de plectres...

Il s'agit d'une notation pratique et lisible, bien qu'incomplète au niveau des effets de timbres. 
En effet, il n'y a qu'à écouter avec une oreille électroacoustique la musique de guitare électrique pour voir au-delà, y compris dans l'univers musical populaire, Jimi Hendrix est justement emblématique à cet égard. En apparence strictement blues-rock, ce musicien a indéniablement effectué un travail électroacoustique, son versant expérimental rendu acceptable au grand public par l'étiquette "psychédélique ». Cela n’a pas échappé aux compositeurs « savants » depuis 1970.

\section{2. Musiques de timbres et notations}

Considérons maintenant des langages plus « sophistiqués ». Comment noter la musique de guitare électrique avec tous ses effets idiomatiques et de timbres, avec le degré de précision et de liberté attendu des compositeurs savants contemporains?

La partition suivante ${ }^{17}$ illustre les capacités d'une notation solfégique étendue par quelques effets de timbres (fig. 11) :

Figure 11 : notation contemporaine pour guitares

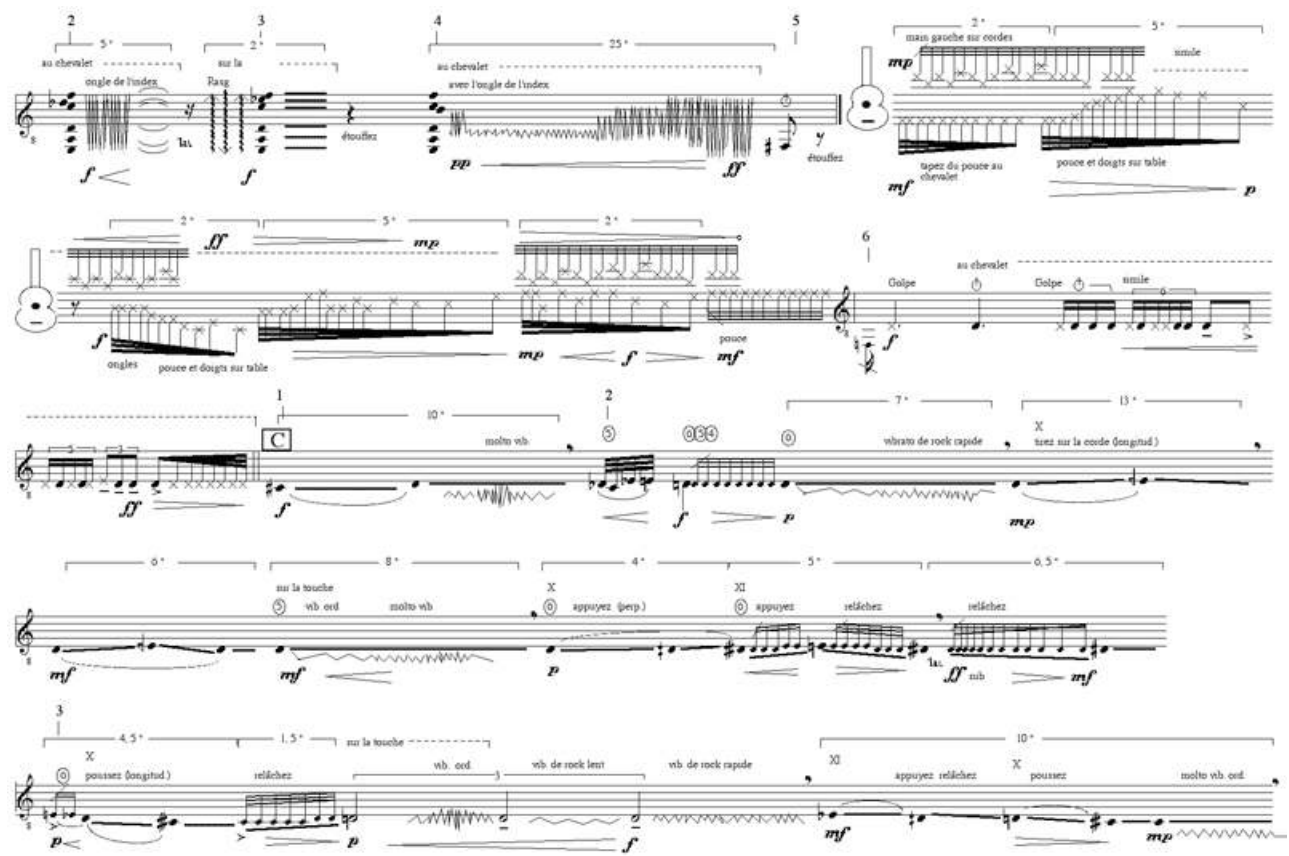

On peut remarquer ici un tempo libre, un jeu en trémolos, frottés, bruits et pizzicatos ó, une polyphonie de gestes, divers ornements et micro-intervalles, etc. Pour éclaircir certains graphismes, des indications verbales sont ajoutées. Les numéros correspondent aux changements de programmes des multi-effets. Il s'agit toutefois d'une partition pour toutes guitares, un certain nombre de modes de jeux spécifiques à la guitare électrique manquent encore. Voyons un cas plus spécifique.

\section{2. 1. Gestes spécifiques}

39 La partition suivante, dédiée à Claude Pavy, a été strictement écrite pour guitare électrique, multi-effets et pédalier numériques comportant des commutateurs, banques de sons et deux pédales continues (volume et dosage d'effet) ${ }^{18}$ : 
Figure 12 : pièce pour guitare électrique

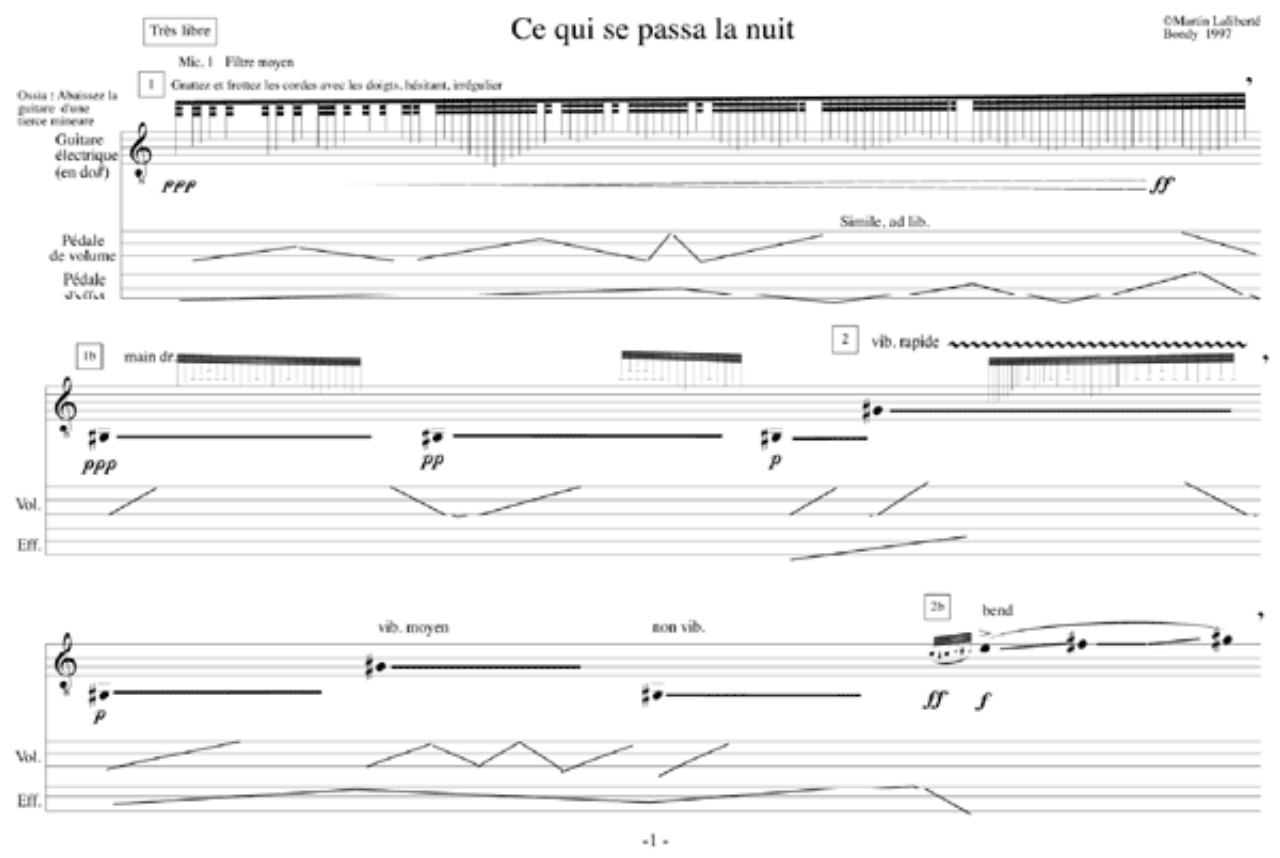

40 L'objectif est ici de noter les gestes de contrôles du guitariste électrique, en plus des notes, rythmes et commutateurs habituels, mais avec dépouillement et clarté. La distinction de la force du geste (notation dynamique usuelle) et le volume final ( $2^{\mathrm{e}}$ portée) est rendue nécessaire par le lent crescendo global avec des accidents locaux. La $3^{e}$ portée note le mouvement de la pédale d'effet, contrôlant à la fois la quantité de modulation en anneau et la profondeur de la réverbération. Remarquez enfin les variantes de vibratos.

41 La page suivante est plus chargée et ajoute d'autres gestes, de même que la tige de vibrato (whammy bar) produisant une sonorité distincte du vibrato de main gauche : 
Figure 13 : Ce qui... la nuit, $2^{\mathrm{e}}$ page

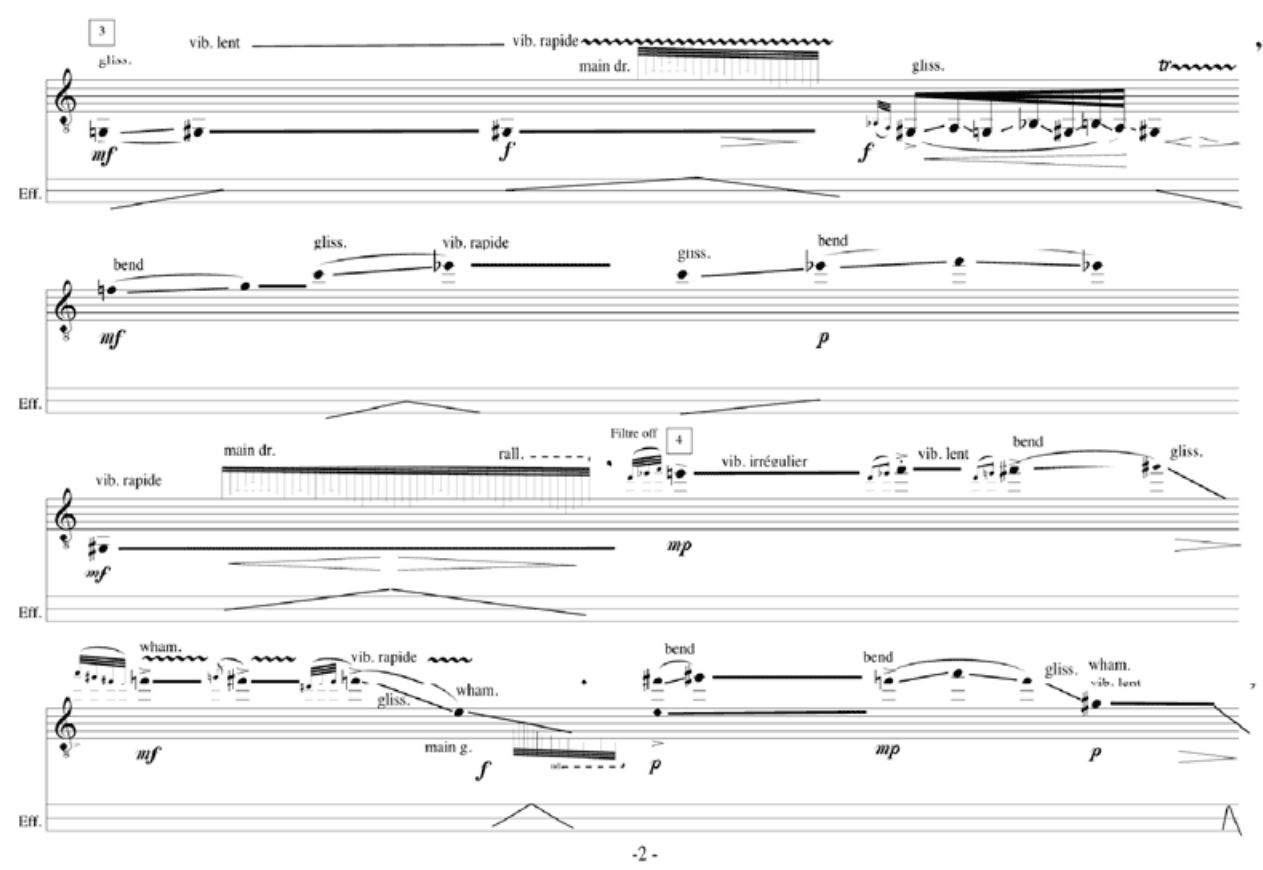

42 Comme les effets de volume ne sont plus distincts de la force des gestes, la $2^{\mathrm{e}}$ portée a disparu. La page suivante introduit une notation des accords et développe les mouvements de whammy:

Figure 14 : Ce qui... la nuit, $3^{\mathrm{e}}$ page

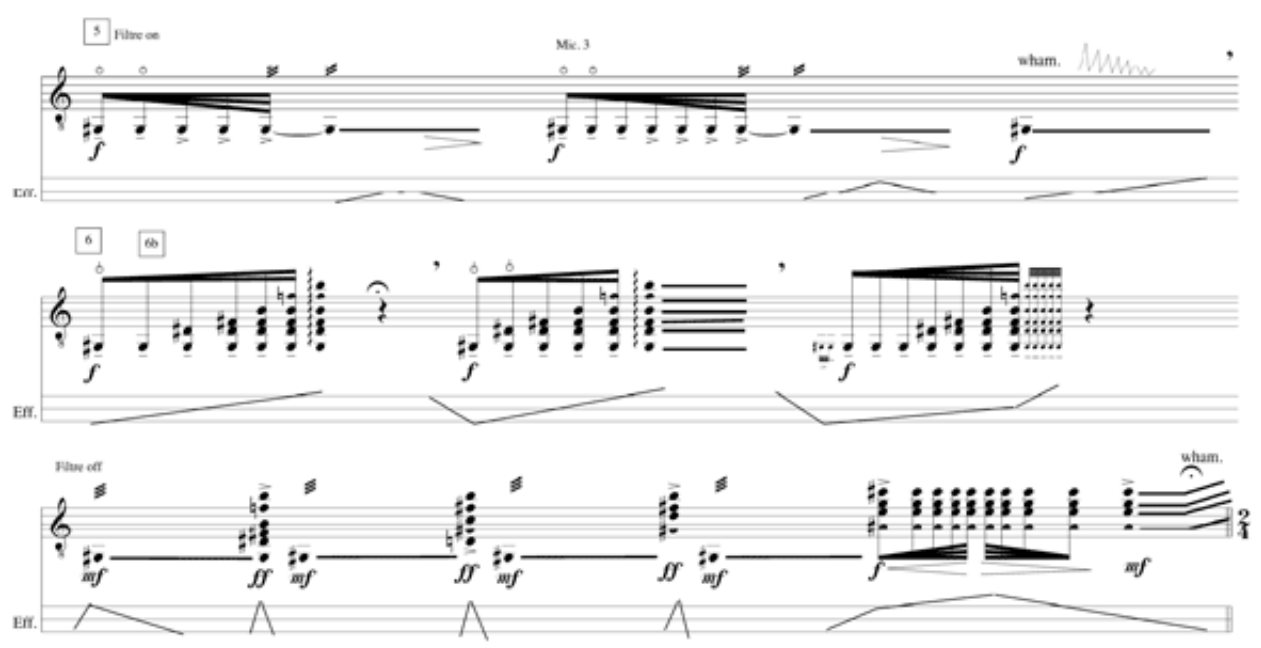

Cette notation a été élaborée en synthétisant de nombreuses expérimentations dans la notation du timbre depuis 1960, en plus de conseils de Claude Pavy. Les aspects les plus courants ont été étudiés dans l'ouvrage indispensable de Kurt Stone ${ }^{19}$, synthèse du colloque de l'UNESCO sur la notion musicale, à Gand en 1974. D'autre part le lecteur assidu de partitions contemporaines peut effectuer sa propre synthèse. Il se dégage de cette enquête une série d'usages efficaces. 


\section{2. 2. Usages courants}

On peut en réalité retrouver différentes pratiques complémentaires :

- noter les gestes mélodico-rythmiques prescriptifs avec notation musicale étendue ;

- noter les gestes semi improvisés (boîtes ou ad lib) ;

- noter les bascules de commutateurs ;

- noter les transitions de timbres ;

- noter des niveaux relatifs ;

- noter exactement.

Les exemples 12-14 illustrent les premier et second cas. Pour le $3^{\mathrm{e}}$, Stone et bien d'autres proposent de noter simplement les changements ponctuels (fig. 15) ${ }^{20}$ :

Figure $15:$ commutateurs ponctuels

\section{Distorsion}

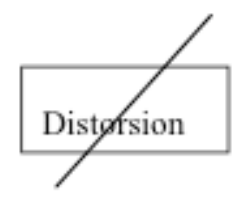

Programme 3 Programme 4

Une notation ponctuelle de ce type est particulièrement adaptée à des effets discontinus ou momentanés, des effets percussifs, dans la perspective que je propose.

Les mêmes proposent de noter les transitions de timbres, - les effets vocaux au sens où je l'entends - avec différents degrés de précision, en fonction de l'exactitude souhaitée (fig. 16) :

Figure 16 : différents degrés de précision
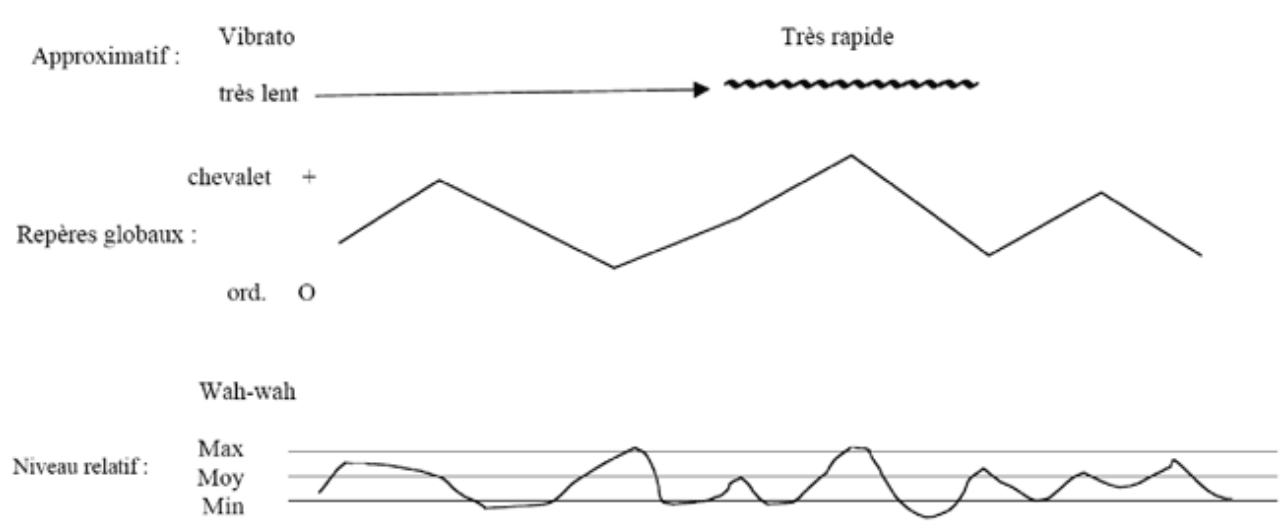

Exact :

L'objectif de ces degrés variables de précision est principalement de maintenir une notation suffisante mais pas superflue. Le second objectif est de demeurer compatible avec la notation solfégique, comme on l'a vu dans les exemples 12 à 14 . Les notes, rythmes et transitions de timbres sont notés tandis que les chiffres indiquent les numéros de programmes que le guitariste appelle tour à tour sur son pédalier. 
49 Si les gestes mélodico-rythmiques ou de contrôle du timbre sont alors correctement notés, il faut remarquer que la sonorité produite n'est encore que partiellement lisible. En effet, les mutations sonores par le biais des traitements sont masquées, cachées derrière les discrets numéros de programme.

\section{2. 3. Programmes} spécificité d'un matériel local et l'obsolescence rapide du matériel courant. L'étude des
partitions propose encore quelques pistes pratiques, encore à des degrés de précision
variable:

- descriptions suggestives ;

- descriptions générales ;

- descriptions spécifiques.

52 Voici quelques descriptions issues de la notice de la pièce ZLOM de Vinko Globokar ${ }^{21}$ : les commentaires pour le synthétiseur ouvrent une autre piste intéressante. C'est une description suggestive :

Synthétiseur

Inventer des programmes en fonction des mots («comme un claquement des mains », « comme un miaulement »...).

Dans les faits, cependant, le compositeur utilise aussi des graphismes suggestifs sur portée à trois lignes et indications suggestives, ce qui est une pratique assez usuelle depuis le début des années 1960 (fig. 17):

Figure 17 : Globokar : Zlom

\section{Imiter le timbre des instruments de percussions}

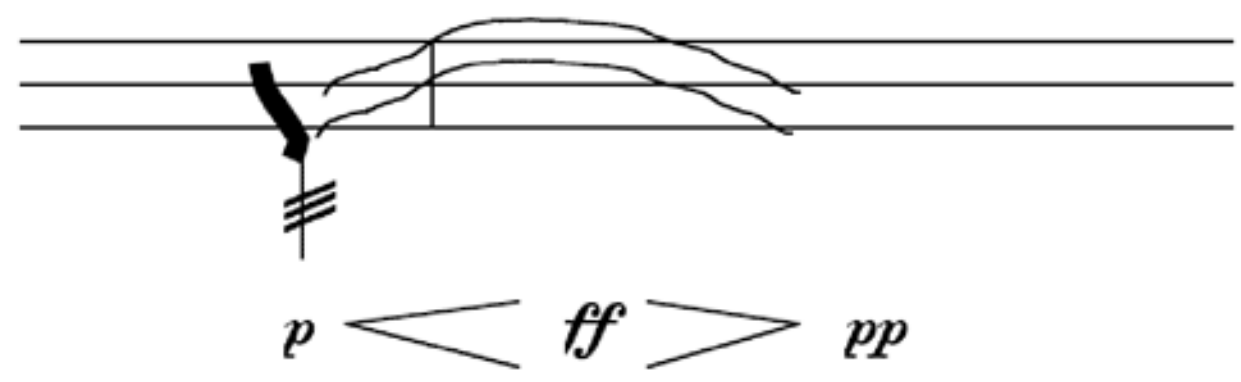

54 Cet exemple combine indication suggestive et notation graphique à repères approximatifs de hauteur. Une autre indication de la notice est plus précise :

Guitare électrique

Le guitariste doit disposer d'appareils de transformation et de saturation du son ainsi que d'une pédale d'amplification ${ }^{22}$. Ensuite, le compositeur précise deux réglages plus spécifiques pour les effets de guitare mais encore assez généraux :

Flanger 1 : son légèrement déformé

Flanger 2 : son déformé ${ }^{23}$ 
Cela peut paraître approximatif, mais cette façon de faire laisse une place à la créativité du musicien, à une adaptation à l'interprétation particulière de l'ensemble, à l'acoustique de la salle, etc. C'est une solution pragmatique et efficace qui ne diffère pas tellement de celles concernant les percussions ou les archets, au fond.

Pour sa part, Hugues Dufourt propose dans ses pièces avec guitare électrique, comme L'̂le Sonnante $e^{24}$ une notation assez spécifique, après une description détaillée du matériel employé lors de la création de la pièce ${ }^{25}$ :

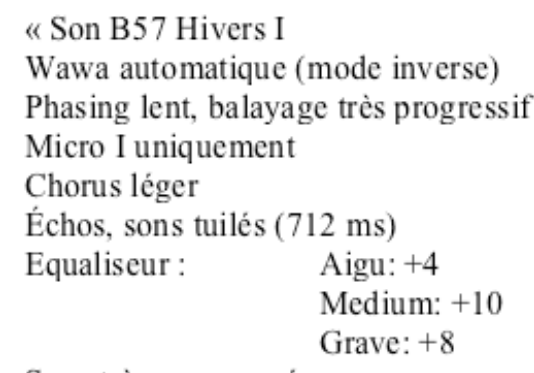

Sons très compressés ”

\author{
Son B66 Île sonnante III \\ Compresseur léger \\ Distorsion douce \\ Echos $950 \mathrm{~ms}$ à $1 \mathrm{sec}$ \\ écho aussi fort que son direct \\ Chorus léger \\ Modulation en anneau : \\ $1 / 3 \bmod ., 2 / 3$ direct \\ Porteuse à $l a \neq 3$
}

58 Le tout se complète d'indications de jeu dans la partition et parfois de graphismes: nuances, mouvements de pédales, etc. Une telle description est à la fois utilement précise et problématique en cas d'obsolescence du matériel. Par exemple, que signifie «EQ aigu + 4 » hors de la pédale Boss d'origine ? Par ailleurs, ces indications ne donnent pas l'ordre des branchements des modules, ce qui peut avoir beaucoup d'incidence sur le résultat. Enfin, ce matériel analogique pose problème, si on espère la survie dans le temps de ces compositions et leur inscription au répertoire stabilisé, au-delà de la durée de vie du matériel commercial courant.

\section{2. 4. Standardisation et pédales}

Après une période inventive très diversifiée, 1960-1980 (Dunlop, Mu-Tron, Electoharmonix, Arbiter, MXR...), les années 1980 ont vu disparaître de nombreux fabricants de pédales d'effets et la large diffusion d'un petit nombre de marques. Les pédales Boss ont alors largement dominé le marché, ce qui a permis une première forme de standardisation $d u$ traitement $d u$ son, après la stabilisation de l'amplification ${ }^{26}$. De tels commentaires sont devenus possibles :

Purple Haze, Album : 'Are You Experienced ?'

Une composition géniale qui a tellement compté pour la guitare et la musique en général. Comment une telle magie a-t-elle pu émaner d'un son de guitare ? Le fait de dénicher une vieille fuzz au Germanium vous aidera. Sinon : drive à $80 \%$, level à $40 \%$ et peu de médiums. Pour la magie, désolé, on ne l'achète pas dans un magasin de musique $!^{27}$.

Les indications de réglages sont ici tout à fait ceux des pédales de la marque Boss. Ces pédales sont en général spécialisées dans quatre familles d'effets :

- Effets de hauteur : octaveur, harmoniseur, vibrato...

- Effets d'amplitude : compresseur, sustainer, tremolo, wah-wah automatique, distorsions et overdrives...

- Effets temporels : délais, réverbérations, phasing, flanging, chorus...

- Effets de spectre: wah-wah, filtres fixes (EQ), distorsions et overdrives, phasing, flanging, chorus, modulateur en anneau... 
61 C'est ce type de matériel courant que les compositeurs savants des années 1970-1990 et leurs guitaristes ont utilisé, comme Dufourt ou Globokar. Ces pédales constituent d'ailleurs une variante guitaristique de synthétiseur modulaire analogique. Les réglages de ces pédales demeurent le plus souvent fixes, à cause d'une ergonomie rudimentaire et mal adaptée au jeu des guitaristes (petits boutons peu accessibles, au sol). On se restreint habituellement ${ }^{28}$ aux pédales préréglées avec commutateur on/off, sauf pour la wah-wah et le volume, qui sont des pédales d'expression continues (fig. 18) ${ }^{29}$ :

Figure 18 : pédalier analogique

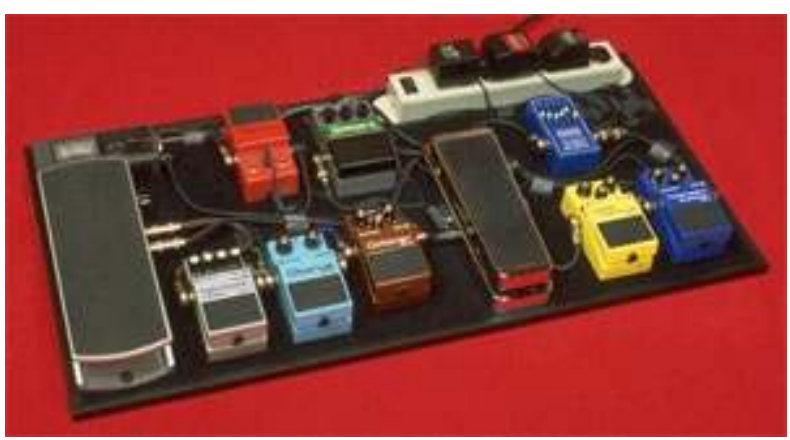

Comme pour l'informatique musicale dans son ensemble, des reproductions virtuelles de ces effets apparaissent depuis une dizaine d'années, ce qui permet même de trouver certaines « espèces disparues » (fig. 19) ${ }^{30}$ :

Figure 19 : quelques « classiques » retrouvés

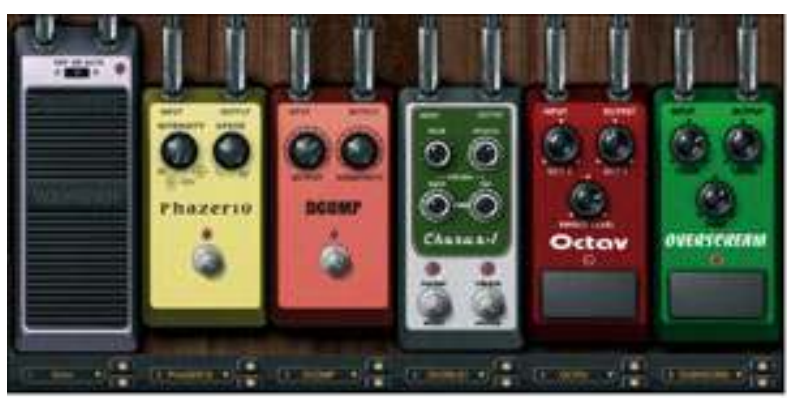

Il s'agit d'une tentative, louable, de standardisation et de lutte contre la disparition du matériel analogique des années 1960-1980, de même qu'un projet commercial d'imposer le rééquipement. Ces plug-in historicistes sont généralement pilotés par des pédaliers numériques, reproduisant la logique des pédaliers analogiques, avec l'ergonomie et l'ouverture informatiques en plus : (fig. 20) :

Figure 20 : pédalier MIDI

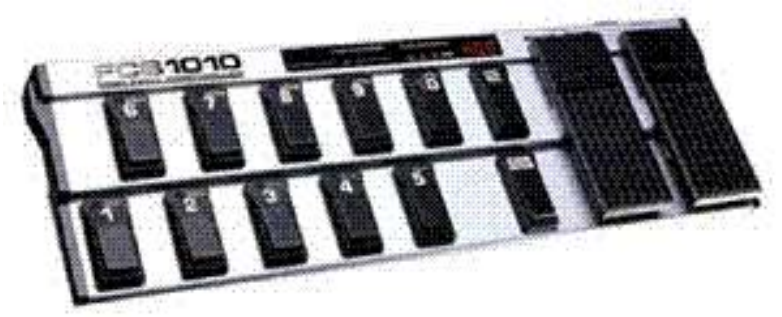


64 wah-wah) et d'une pédale de volume. Les modifications de réglages se font principalement par changement de programme (regroupement par banques et numéros), ce qui permet de bien compenser la faible ergonomie initiale. Cela dit, un tel matériel n'est que partiellement traditionnel; de nombreux exemples de détournements sont bien connus depuis les années $1990^{31}$. On peut par exemple réassigner à une large gamme de paramètres sonores les mouvements de la pédale d'expression.

\section{2. 5. Développement des interfaces}

Si une standardisation relative du matériel et l'élaboration d'une sorte de répertoire commun de gestes musicaux et de modèles sonores a permis aux compositeurs de construire la musique de guitare électrique récente, les développements ne sont probablement pas encore terminés. En effet, le matériel informatique aujourd'hui courant permettrait d'élargir considérablement les gestes possibles (fig. 21) :

Figure 21 : typologie de contrôleurs

\begin{tabular}{|c|c|c|}
\hline Type & Plage valeurs & Commentaires \\
\hline $\begin{array}{l}\text { Bouton simple } \\
=\text { commutateur }\end{array}$ & $0 / 1$ & $\begin{array}{l}\text { Peu sensible mais efficace et peu coûteux } \\
\text { Clairement percussif (pauvre en information) mais } \\
\text { efficace rythmiquement }\end{array}$ \\
\hline $\begin{array}{l}\text { Boutons } \\
\text { dynamiques (pad) }\end{array}$ & $0-127$ & $\begin{array}{l}\text { Type boîte à rythme ou clavier musical } \\
\text { Percussif + riche } \\
\text { Peu usité sur guitares (vieux modèle Roland) }\end{array}$ \\
\hline $\begin{array}{l}\text { Potentiomètres } \\
\text { rotatifs }\end{array}$ & $\begin{array}{l}0-127,0-256 \\
\text { Centré }(+/-)\end{array}$ & $\begin{array}{l}\text { Mouvements de poignet ou de doigts (finesse) } \\
\text { Plus vocal }\end{array}$ \\
\hline Curseur linéaire & $\begin{array}{l}0-127,0-255,0-511 \\
\text { Centré (+/-) } \\
\text { Réponse linéaire vs } \\
\text { logarithmique } \\
\text { Horizontal ou vertical } \\
\end{array}$ & $\begin{array}{l}\text { Effet variable selon les paramètres à contrôler } \\
\text { Vocal } \\
\text { Rare sur guitares }\end{array}$ \\
\hline Pédale simple & $0 / 1$ & Déclencheur pour pied (percussif) \\
\hline Pédale continue & $\begin{array}{l}0-127 \\
\text { Centrée }(+/-)\end{array}$ & $\begin{array}{l}\text { Contrôle dynamique (vocal) } \\
\text { Efficacité dépend de façon critique de la qualité de } \\
\text { la courbe de réponse (cf Ernie Ball vs pédale bon } \\
\text { marché) }\end{array}$ \\
\hline Molette continue & $0-127$ & $\begin{array}{l}\text { Contrôles variables (vocal) } \\
\text { Sur la guitare MIDI }\end{array}$ \\
\hline Molette crantée & $+1-$ & Sensibilité des pas et mouvement rotatif \\
\hline Molette centrée & $\begin{array}{l}0-127 \\
-8000 /+8000\end{array}$ & $\begin{array}{l}\text { Revient au centre } \\
\text { Type pitch bend (meilleure résolution } 14 \text { bits) }\end{array}$ \\
\hline $\begin{array}{l}\text { Whammy réel ou } \\
\text { virtuel }\end{array}$ & $0-127$ ou plus & Très vocal \\
\hline
\end{tabular}

Ces interfaces étendues pourraient être montées sur la guitare électrique et utilisées de façon ergonomique et idiomatique, comme l'ont montré les présentations des autres participants du colloque. On peut déplorer le conservatisme des fabricants industriels de la guitare à ce niveau, qui n'utilisent guère que les boutons inventés dans les années 1940. Certains modèles de guitare-synthétiseur de Roland - la G-808 et son GR-300, en particulier ${ }^{32}$ - échappaient à cette frilosité, mais ont aujourd'hui disparu. L'interface GK-3 actuelle ne conserve qu'une partie des contrôleurs très ergonomiques de ses meilleurs prédécesseurs. 


\section{Conclusion}

67 La guitare électrique a trouvé sa seconde maturité avec Jimi Hendrix, ce qui lui a permis d'attirer de nombreux compositeurs de la tradition savante. 40 ans après Woodstock, la notation pour guitare électrique s'est développée et a elle aussi peut-être atteint une certaine maturité, avant la prochaine vague de mutations.

\section{BIBLIOGRAPHIE}

Barkati Karim, Entre temps réel et temps différé, thèse de doctorat, Saint-Denis, université de Paris 8, 2009, http://karim.barkati.online.fr/Universite/Doctorat/These/VersionFinale.

Egraz Ludovic, Les plus grands plans du rock, Paris, Guitare \& claviers (Carnet de notes), mars 1995.

Laliberté Martin, « Archétypes et paradoxes des nouveaux instruments de musique », in Les nouveaux gestes de la musique, R. de Vivo, H. Genevois (éd.), Marseille, Parenthèses, 1999,

p. 121-138.

Laliberté Martin, "Origines et devenir des "nouvelles technologies musicales" », in Musiques, arts et technologies : pour une approche critique, R. Barbanti, E. Lynch, C. Pardo et M. Solomos (éd.), Paris, l'Harmattan (Musique-Philosophie), 2004, p. 347-360.

Laliberté Martin, Archétypes instrumentaux, analyse et composition musicale. Bilans et prospective, mémoire d'Habilitation à Diriger des recherches, Horacio Vaggione (dir.), université de Paris 8 , 3 volumes et deux cd, 2006, 349 pages.

Murray Charles Shaar, Jimi Hendrix vie et légende, Paris, Seuil (Points), 1989/1993, 351 pages.

Schaeffner André, Origines des instruments de musique, Paris, Mouton/Maison des sciences de l'Homme (Rééditions), 2/1980.

\section{NOTES}

1. Voir entre autres Martin Laliberté, «Archétypes et paradoxes des nouveaux instruments de musique", in Les nouveaux gestes de la musique, R. de Vivo, H. Genevois (éd.), Marseille, Parenthèses, 1999, p. 121-138 et " Origines et devenir des "nouvelles technologies musicales" ", in Musiques, arts et technologies: pour une approche critique, R. Barbanti, E. Lynch, C. Pardo et M. Solomos (éd.), Paris, l'Harmattan (Musique-Philosophie), 2004, p. 347-360.

2. En effet, le chant purement vocalique est assez rare; les consonnes sont indispensables au chant dans toute sa richesse, bien entendu.

3. Martin Laliberté, Archétypes instrumentaux, analyse et composition musicale. Bilans et prospective, mémoire d'Habilitation à Diriger des recherches, Horacio Vaggione (dir.), université de Paris 8 , 3 volumes et deux cd, 2006, 349 pages.

4. Ibid. Voir aussi André Schaeffner, Origines des instruments de musique, Paris, Mouton/Maison des sciences de l'Homme (Rééditions), 2/1980, p. 371-377. 
5. Il rejoint certaines actions spectaculaires du guitariste, par exemple celle de l'immolation de sa guitare à Monterey en 1967 ou des déclarations du musicien se présentant plus comme un « entertainer» qu'un «chanteur ». Voir l'excellente biographie de Charles Shaar Murray, Jimi Hendrix vie et légende, Paris, Seuil (Points), 1989/1993, 351 pages.

6. Artie Traum, Arti Funaro, Improvising rock guitar, New York, AMSCO, 1983, p. 32.

7. Cette transcription rudimentaire date d'une époque sans doute encore un peu dilettante mais se destine surtout à un public débutant dans un ouvrage couvrant un large spectre musical.

8. À ce point-ci de la communication, un débat intéressant s'est amorcé avec le guitariste Claude Pavy. Celui-ci maintient que la tablature est un outil «pour les amateurs ne connaissant pas le manche de leur instrument ». Il privilégie fortement la notation solfégique, ce qui a du poids venant d'un guitariste de talent et d'un pédagogue expérimenté. D'autres guitaristes présents se montraient toutefois plus nuancés à cet égard, soulignant la clarté dans certains cas de la tablature, en particulier pour la délicate question de la sonorité spécifique de chaque corde ou d'autres effets de timbres. On peut par ailleurs ajouter que la notation solfégique permet une meilleure précision rythmique et une clarté polyphonique qui dépasse les capacités de la tablature.

9. Ludovic Egraz, Les plus grands plans du rock, Paris, Guitare \& claviers (Carnet de notes), mars 1995 , p. 24.

10. Dave Whitehill et al., Radio One, Bella Godiva/Hal Leonard,1991, p. 147.

11. Il s'agit encore de la transcription de la version du cd Rykodisc « Radio One », 1967.

12. Ibid., p. 120.

13. Ibid., p. 121

14. Sans parler des franches erreurs de transcriptions d'éditions moins scrupuleuses, notamment au niveau rythmique (la méconnaissance des rythmes irrationnels de certains transcripteurs joue souvent des tours) ou harmonique (la théorie musicale est parfois un peu négligée).

15. Ludovic Egraz, Les plus grands plans du rock, p. 21.

16. Dave Whitehill, Radio One, p. 284.

17. Martin Laliberté, Le sommeil de Protée, pour guitare et ordinateur, 1990.

18. Martin Laliberté, Ce qui se passa la nuit, pour guitare électrique, commande du Festival Why Note, 1997.

19. Kurt Stone, Music notation in the $\mathrm{XX}^{\text {th }}$ century, New York, Norton 1982.

20. Ce sont des exemples tirés des partitions de Hugues Dufourt, notamment.

21. Ricordi, 1997.

22. Ibid.

23. Ibid.

24. Salabert, 1990.

25. Ibid.

26. Fender, Marshall...

27. Ludovic Egraz, Les plus grands plans du rock, Introduction de Purple Haze, p. 21.

28. De nombreux guitaristes plus aventureux, comme Robert Fripp ou Steve Hackett disposaient leurs pédales tout autrement, sur des tables ou dans des racks surélevés, dès les années 1970.

29. http://www.stompin-ground.com/products/sgproduct-cat.htm, consulté en septembre 2009.

30. Plug-ins Amplitube pour bancs de montage audionumérique, http://www.ikmultimedia.com/ Main.html?guitarbasssoftware/index.php, consulté en juin 2009.

31. Voir la thèse de Karim Barkati, Entre temps réel et temps différé, Saint-Denis, université de Paris 8, 2009, http://karim.barkati.online.fr/Universite/Doctorat/These/VersionFinale.

32. Voir http://www.joness.com/gr300/GR-300.htm. Consulté en septembre 2009. 
INDEX

Mots-clés : archétypes instrumentaux, composition contemporaine, geste, notation musicale Personnes citées : Hendrix (Jimi)

\section{AUTEUR}

\section{MARTIN LALIBERTÉ}

Université de Paris-Est, laboratoire Littératures, Savoir et Arts EA 4120, équipe CCAMAN 